\title{
S-wave $\pi$-Nucleus Repulsion and Dirac Phenomenology
}

\author{
S. Chakravarti \\ Department of Physics, California State Polytechnic University, Pomona, CA 91768 , \\ $U S A$ \\ B.K. Jennings \\ TRIUMF, 4004 Wesbrook Mall, Vancouver, B.C. V6T 2A3, Canada
}

\begin{abstract}
A relativistic $\pi$-nucleon potential is extended to $m^{*} \neq m$ to investigate the possibility of generating s-wave $\pi$-nucleus repulsion. We find that relativity does indeed generate significant repulsion, the exact amount depending on the details of the calculation. In contradistinction the $t \rho$ approximation gives very little repulsion.
\end{abstract}

Submitted to Physics Letters

July 18, 2018 
The experimental data on low-energy pion-nuclear interactions [四] from a variety of sources, e.g., pionic atoms at zero pion kinetic energy $E_{\text {kin }}$, elastic, inelastic and charge exchange reactions at $E_{\text {kin }} \leq 50 \mathrm{MeV}$, etc., are generally well described by theoretically motivated phenomenological optical potentials [2]. These potentials are generated by using the nonrelativistic impulse approximation plus density dependent corrections. Unfortunately they fail to produce the central pion-nucleus repulsion of about $30 \mathrm{MeV}$ required by fits of the experimental data [3].

The underlying isoscalar $\pi \mathrm{N}$ s-wave interaction is exceptionally small at threshold (with a scattering length $b_{0}=-0.010 m_{\pi}^{-1}, m_{\pi}$ being the pion mass), its double-scattering modification [4] is relatively ineffective, and the dispersive contribution accompanying the imaginary $\pi \mathrm{NN}$ absorption term is believed to be attractive [5]; if this latter contribution were to blame, it would have to be repulsive, with a magnitude of up to five times its imaginary counterpart.

Recently there has been considerable controversy over the role of relativity in addressing this problem. Birbrair and Gridnev [6, 7] have made the observation that, to the extent that Nuclear Dirac Phenomenology provides a valid and meaningful description of the gross properties of nuclei at low energies, it naturally offers additional s-wave repulsion.

This approach has been attacked from two fronts. First, it has been shown that for pion scattering there are large off-shell ambiguities [8]. Second, it has been argued that for pions the impulse approximation is not valid, since using $m^{*}$ internally in diagrams cancels most of the $m^{*}$ effects coming from the impulse approximation [9, 10]. These are both valid concerns.

One way to address these concerns is to take a relativistic model that describes the free $\pi$-nucleon scattering and examine its prediction when the nucleon effective mass $m^{*}$ is varied. The off-shell behavior is then fixed by the model and is no longer ambiguous. In addition $m^{*}$ is included in all possible places and one goes beyond the impulse approximation. In this letter we use the relativistic $\pi$-nucleon potential developed by Pearce and 
Jennings [11] to carry out this program. We find that there is a repulsion on the order of a few tens of $\mathrm{MeV}$ coming from the relativistic effects. However, we also find that both objections to the work of Birbrair and Gridnev are correct. The $t \rho$ approximation gives very little dependence on $m^{*}$ while a significant effect arises from putting $m^{*}$ in intermediate propagators.

The present work is a first step towards developing a full relativistic optical potential. That work would require the consideration of other partial waves and the treatment of Pauli blocking. A relativistic potential is interesting in its own right and, as pointed out in ref. [12], is crucial in reactions like $\left(\mathrm{p}, \mathrm{n} \pi^{+}\right)$where the nucleons are treated most easily in a Dirac approach.

An early attempt to develop a relativistic model was made by Miller and Noble [13]. They used the $\sigma-\omega$ model that did not attempt to simultaneously describe the free nucleon-nucleon scattering. Thus it is quite different from our approach.

An alternate approach to the relativistic pion-nuclear problem has been developed by Leisi and co-workers [14]. This approach introduces an explicit scalar field $(\sigma)$ for the pion to couple to. In our work we are only concerned with the effects of the pion coupling to an in-medium nucleon and have nothing to say, at the present time, about an explicitly enhanced $\sigma$ field.

We start with the $\pi$-nucleon potential developed by Pearce and Jennings [11]. That potential includes $\rho, \sigma$ exchange and the nucleon and delta pole and crossed-pole terms. The nucleons are treated as Dirac particles and negative energy propagators are included at each step of the process. In the present work we simply take the Pearce-Jennings model and replace $m$ with $m^{*}$. In particular the mass appears in the energy denominators of the nucleon and delta pole and crossed-pole terms and in the free spinors. One has to be careful not to replace $m$ where it has a purely formal role such as in the definition of the pseudo-vector coupling. We have used the smooth propagator [15] and have checked that our results agree with ref. [11] when $m^{*}=m$.

The calculation of phase shifts uses bare values for the nucleon and delta masses and 
couplings of the pole diagrams in the $P_{11}$ and $P_{33}$ channels. These are calculated by the renormalisation procedure described in ref. [16] wherein the $P_{11}$ amplitude is required to have a pole at the physical nucleon mass $m$, and the residue is required to reproduce the physical $\pi$ NN coupling constant. The bare delta mass and coupling constant are treated as parameters and adjusted to fit the experimental phase shifts.

The values to use for the in-medium $\pi \mathrm{NN}$ and $\pi \mathrm{N} \Delta$ couplings are ambiguous. The obvious thing to do is to keep the bare couplings fixed. The renomalisation will then occur due to the dressing generated by solving the Lippmann-Schwinger equation. This will work well in the p-waves where the renormalisation is generated directly in this manner in free space. In this case the physical couplings will vary with the effective mass $m^{*}$.

For the s-waves the physical couplings are put in by hand rather than being generated within the model. It is not clear how they will change in the medium. A possible constraint arises from the Goldberger-Treiman relation. It has been suggested that inmedium we have two Goldberger-Treiman relations [17] depending on which quantum numbers we are considering. For the s-wave the renormalisation is different from that for the p-waves and is quite small [17]. This suggests that one keep the couplings fixed at their physical values. We will give results for both cases.

In our work we chose $m^{*}$ as the (variable) physical nucleon mass and obtained the corresponding bare nucleon mass by the renormalisation procedure. If we keep the bare coupling constants fixed at the value for $m^{*}=m$ the renomalised values vary with $m^{*}$. The values of the dressed coupling constant and the bare nucleon mass obtained this way are shown in table 1.

To obtain the dressed delta parameters as a function of $m^{*}$, we set the dressed deltanucleon mass difference $m_{\Delta}^{*}-m^{*}$ equal to the physical mass difference. Calculations were then performed for the $P_{33}$ channel at energies around resonance $\left(\delta_{P_{33}}=90^{\circ}\right)$. The value of the dressed delta mass was then extracted from the location of the resonance, and that of the dressed coupling constant $f_{\pi N \Delta}$ from the slope of the phase shift curve as a 
function of energy. Values of the extracted parameters are shown in table 2.

The results for the scattering lengths in each isospin channel are given in table 3 for three cases: fixed bare couplings, fixed physical couplings and the $t \rho$ approximation. For each case, we also give the isoscalar pseudopotentials 18 obtained from the scattering lengths, using a nuclear matter density of $0.17 \mathrm{fm}^{-3}$.

It is instructive to examine, for a fixed pion energy, the contributions to the on-shell Born term [11] from the individual diagrams in each isospin channel as a function of $m^{*}$. These are shown in figs. 1 and 2 for the case of fixed bare couplings, with $T_{\pi}=20 \mathrm{MeV}$. We see that the main variation is in the pole and crossed-pole terms. The variation has two main sources. First, the energy denominator varies approximately as $1 / m^{*}$. Second, the couplings vary (when we keep the bare couplings fixed).

Next, we note that in the $t \rho$ approximation there is very little $m^{*}$ dependence. This is consistent with the off-shell extrapolation used by Gal et al. [8] but inconsistent with the extrapolation of Birbrair et al. [6, 7]. Thus we conclude that the $t \rho$ approximation cannot account for the repulsion.

We see, however, that including $m^{*}$ also in the interior of diagrams does give repulsion. This comes about from the energy denominators of the z-graphs. The most repulsion arises when we keep the bare coupling constants fixed and gives about $20 \mathrm{MeV}$ repulsion at nuclear matter density. When the dressed couplings are kept fixed at their physical values (which we believe is more realistic) the repulsion is cut almost in half. Thus we do not have enough repulsion to cure the entire problem but the effect is in the correct direction and within a factor of 2 of the correct size.

In summary, we have examined the predictions of a relativistic $\pi$-nucleon potential when $m^{*} \neq m$ for two different choices of the in-medium coupling constants, and compared them with that of the $t \rho$ approximation. We have found that relativity does indeed generate significant repulsion, the exact amount depending on the choice of the in-medium couplings. In contradistinction the $t \rho$ approximation is found to give very little repulsion. 
S.C. gratefully acknowledges the hospitality of the TRIUMF Theory group where this work was begun. The authors acknowledge the hospitality of the National Institute of Nuclear Theory, University of Washington, where part of this work was carried out. B.K.J. acknowledges financial support from the Natural Sciences and Engineering Council of Canada.

\section{References}

[1] W. Kluge, Rep. Prog. Phys. 54 (1991)1251.

[2] M. Ericson and T.E.O. Ericson, Ann. Phys. 36 (1966) 323; J.A. Carr, H. McManus and K. Stricker-Bauer, Phys. Rev. C25 (1982) 952 and earlier works listed there; R. Seki and K. Masutani, Phys. Rev. C27 (1983) 2799; O. Meirav et al., Phys. Rev. C40 (1989) 843.

[3] J.G.J. Olivier, M. Thies and J.H. Koch, Nucl. Phys. A429 (1984) 477; J. Konijn, C.T.A.M. de Laat, A. Taal and J.H. Koch, Nucl. Phys. A519 (1990) 773.

[4] J. Hüfner, Phys. Rep. 21 (1975) 1.

[5] C. Garciá-Recio, E. Oset and L.L. Salcedo, Phys. Rev. C37 (1988) 194 and earlier references listed there.

[6] B.L. Birbrair and A.B. Gridnev, Nucl. Phys. A528 (1991) 647.

[7] B.L. Birbrair, A.B. Gridnev, L.P. Lapina, A.A. Petrunin and A.I. Smirnov, LowEnergy Pion-Nucleus Interaction and Nuclear Relativism, LNPI preprint 1677 (March 1991).

[8] A. Gal, B.K. Jennings and E. Friedman, Phys. Lett. B281 (1992)11.

[9] V. Koch, E. Oset, Nucl. Phys. A541 (1992) 578-584.

[10] E. Oset, private communication. 
[11] B.C. Pearce and B.K. Jennings, Nuc. Phys. A528 (1991) 655.

[12] E.D. Cooper, K. Hicks and B.K. Jennings, Nucl. Phys. A470 (1987) 523.

[13] G.A. Miller and J.V. Noble, Phys. Rev. C21 (1980) 2519.

[14] P.F.A. Goudsmit, H.J. Leisi, E. Matsinos, Phys. Lett. B271 (1991) 290;

[15] E.D. Cooper and B.K. Jennings, Nucl. Phys. A483 (1988) 601.

[16] B.C. Pearce and I.R. Afnan, Phys. Rev. C34 (1986) 991.

[17] D.-O. Riska, private communication

[18] T. Ericson and W. Weise, Pions and Nuclei, Clarendon Press, Oxford, 1988, p. 35 


\section{Figure Captions}

Fig. 1. The on-shell Born potential in the S11 channel (solid line) as a function of $m^{*} / m$, for $T_{\pi}=20 \mathrm{MeV}$ and fixed bare couplings. The contributions from the individual diagrams are labelled as follows: (a): nucleon pole; (b): crossed nucleon pole; (c): $\rho$ exchange; (d): $\Delta$ pole; (e): crossed $\Delta$ pole; (f): $\sigma$ exchange.

Fig. 2. Same as in fig. 1 but for the S31 channel. 
Table 1. The values of the dressed $\pi \mathrm{NN}$ coupling constant and the bare nucleon mass for a range of $m^{*}$.

\begin{tabular}{|ccc|}
\hline$m^{*} / m$ & $g_{\pi N N}^{2} /(4 \pi)$ & $\begin{array}{c}m_{o N} \\
(\mathrm{MeV})\end{array}$ \\
\hline 0.5 & 19.85 & 652.4 \\
0.6 & 19.07 & 739.9 \\
0.7 & 18.01 & 827.7 \\
0.8 & 16.79 & 915.8 \\
0.9 & 15.53 & 1004.3 \\
1.0 & 14.30 & 1093.2 \\
1.1 & 13.16 & 1182.7 \\
1.2 & 12.14 & 1272.7 \\
\hline
\end{tabular}

Table 2. Values of the dressed $\pi \mathrm{N} \Delta$ coupling constant and $\Delta$ mass for a range of $m^{*}$.

\begin{tabular}{|cccc|}
\hline$m^{*} / m$ & $f^{2} /(4 \pi)$ & $\begin{array}{c}m_{\Delta}^{*} \\
(\mathrm{MeV})\end{array}$ & $\begin{array}{c}\Gamma_{\Delta} \\
(\mathrm{MeV})\end{array}$ \\
\hline 0.5 & 0.496 & 826.2 & 169 \\
0.6 & 0.538 & 893.1 & 173 \\
0.7 & 0.522 & 968.8 & 161 \\
0.8 & 0.475 & 1052.3 & 144 \\
0.9 & 0.415 & 1140.7 & 128 \\
1.0 & 0.365 & 1231.8 & 113 \\
1.1 & 0.308 & 1324.4 & 102 \\
1.2 & 0.270 & 1417.6 & 92 \\
\hline
\end{tabular}


Table 3. Scattering lengths and pseudopotential strengths for a range of $m^{*}$.

\begin{tabular}{|c|ccc|ccc|ccc|}
\hline & \multicolumn{3}{|c|}{ In Medium } & \multicolumn{3}{c|}{ Free } & \multicolumn{3}{c|}{$t \rho$} \\
& \multicolumn{3}{|c|}{ Couplings } & \multicolumn{3}{c|}{ Couplings } & \multicolumn{3}{c|}{ Approximation } \\
\hline$m^{*} / m$ & $\begin{array}{r}a_{\mathrm{S} 11} \\
(\mathrm{fm})\end{array}$ & $a_{\mathrm{S} 31}$ & $V$ & $a_{\mathrm{S} 11}$ & $a_{\mathrm{S} 31}$ & $V$ & $a_{\mathrm{S} 11}$ & $a_{\mathrm{S} 31}$ & $V$ \\
& $(\mathrm{MeV})$ & $(\mathrm{fm})$ & $(\mathrm{fm})$ & $(\mathrm{MeV})$ & $(\mathrm{fm})$ & $(\mathrm{fm})$ & $(\mathrm{MeV})$ \\
\hline 0.5 & 0.108 & -0.184 & 26.2 & 0.134 & -0.146 & 16.0 & 0.226 & -0.123 & 2.1 \\
0.6 & 0.118 & -0.183 & 25.0 & 0.150 & -0.142 & 13.4 & 0.222 & -0.121 & 2.0 \\
0.7 & 0.140 & -0.169 & 20.1 & 0.167 & -0.136 & 10.6 & 0.218 & -0.119 & 2.0 \\
0.8 & 0.166 & -0.151 & 13.8 & 0.183 & -0.129 & 7.6 & 0.216 & -0.118 & 2.0 \\
0.9 & 0.191 & -0.132 & 7.3 & 0.199 & -0.122 & 4.7 & 0.214 & -0.117 & 2.0 \\
1.0 & 0.213 & -0.116 & 2.0 & 0.213 & -0.116 & 2.0 & 0.213 & -0.116 & 2.0 \\
1.1 & 0.230 & -0.103 & -2.4 & 0.225 & -0.110 & -0.5 & 0.211 & -0.115 & 1.9 \\
1.2 & 0.243 & -0.093 & -5.7 & 0.235 & -0.105 & -2.6 & 0.210 & -0.115 & 1.9 \\
\hline
\end{tabular}

